\title{
Fast and Slow Dynamics in Femtosecond Laser-induced Crack Propagation inside a LiF Single Crystal
}

\author{
Masaaki Sakakura ${ }^{* 1}$, Takuro Okada ${ }^{* 2}$, Yasuhiko Shimotsuma ${ }^{* 2}$, Naoaki Fukuda ${ }^{* 1}$ and Kiyotaka Miura ${ }^{* 2}$ \\ ${ }^{*}$ Office of Society-Academia Collaboration for Innovation, Kyoto Univ., Innovation plaza 204, 1-30 \\ Goryo-ohara, Nishikyo-ku Kyoto, 615-8245, Japan \\ E-mail: msakakura@saci.kyoto-u.ac.jp \\ *2 Dept. of Material Chemistry, Graduate School of Eng., Kyoto Univ., Kyoutodaigaku Katsura, \\ Nishikyo-ku, Kyoto 615-8510, Japan
}

\begin{abstract}
Extension of a crack at a specific location has been observed inside a LiF single crystal after parallel photoexcitation at multiple spots by focused femtosecond (fs) laser pulses. In this study, to elucidate the mechanism of crack extension at a specific location, we observed the dynamics of crack propagation in a LiF single crystal after parallel fs laser irradiation by a pump-probe microscope. The observation showed that there are fast and slow propagation dynamics; the velocity of the faster propagation is comparable to the sound velocity, whereas that of the slow propagation was more than ten times slower than the sound velocity. The slower propagation was observed only in extended cracks. In addition, we observed the transient birefringence distribution during slower propagation (10-24 ns) and found that the birefringence was distributed around normal cracks while there was little strain distribution around extended cracks. Therefore, we interpreted that residual strain, which is the origin of the observed birefringence, in tens nanoseconds could suppress the extension of normal cracks and the slower propagation appeared as a result of the disappearance of the residual strain. DOI: $10.2961 /$ jlmn.2015.03.0015
\end{abstract}

Keywords: Femtosecond laser, dynamics, crystal, crack, dislocation, birefringence, strain

\section{Introduction}

Nonlinear photoexcitation by focused femtosecond (fs) laser pulses can induce local modification in the focal region inside transparent materials [1-7]. The fs laser-induced modification depends on various laser irradiation conditions and material properties. In particular, the morphology of the modification is different between amorphous and crystalline materials. Inside amorphous materials, such as glasses and polymers [1-3], the modification is often localized only in the photoexcited region under weak irradiation condition, and cracks are generated in random directions under strong irradiation condition. On the other hand, inside single crystals [4-6], cracks and dislocations (line defects) are generated in the specific directions (Fig. 1(a)). For example, inside single crystals of rock-salt structures such as $\mathrm{NaCl}$ and $\mathrm{LiF}$, cracks and dislocation bands are generated respectively in the $\langle 100\rangle$ and $\langle 110\rangle$ directions from the photoexcited region $[4,6,8]$. Because such laserinduced cracks and dislocations induce deformation far away from the photoexcited region, they could reduce the uniformity of laser processing in crystals. Therefore, it is important for precise and fine processing of crystals to understand the effect of cracks and dislocations to other laserinduced structural change and the mechanism of crack and dislocation generation.

We have investigated the effect of cracks and dislocations to fs laser induced structural changes in sequential or parallel laser irradiation at multiple spots. In our previous study, we have found that elongation of some cracks at specific locations is suppressed or extended by parallel fs laser irradiation inside a LiF single crystal [8]. Figure 1(b) and 1 (c) show examples of the suppression and extension of crack, respectively. When a fs laser pulse was focused at three points, of which positions are located at the corner of an isosceles triangle of $\theta=75^{\circ}(\theta$ is the vertex angle $)$, the downward crack from the upper photoexcited region was suppressed (Fig. 1(b)). On the other hand, in the case of $\theta=105^{\circ}$, a crack at the same location was extended about (a)

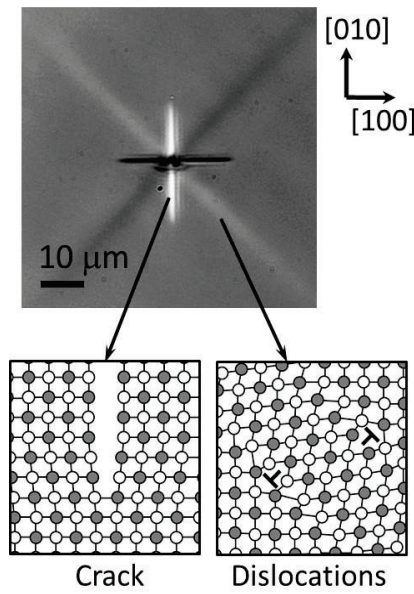

(b) $\theta=75^{\circ}$

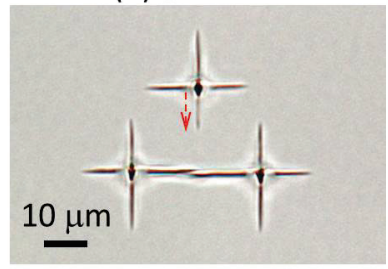

(c) $\theta=105^{\circ}$

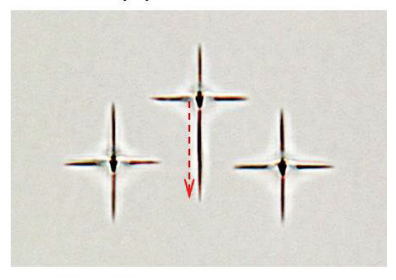

Fig. 1 (a) Polarization microscope image of fs laser induced cracks inside a $\mathrm{LiF}$ single crystal. The photoexcited region is located at the center of the image. Four cracks and dislocation bands were formed in the $\langle 100\rangle$ and $<110\rangle$ directions, respectively. The schematic illustration of lattice deformations due to crack and dislocations were illustrated below the microscope image. (b), (c) Transmission optical microscope images of cracks by parallel fs laser irradiation at three spots inside a $\mathrm{LiF}$ single crystal. 
two times longer than any other cracks. In our first report on these phenomena, we attributed the suppression and extension of the cracks to the modulation of stress distributions due to interference of laser induced stress waves. In fact, we have observed the crack propagation stopped when a compressive stress was generated due to the interference of stress waves at the tip of the crack in the case of Fig. 1 (b) by a time-resolved observation using a pump-probe microscopy [9]. However, according to the time-resolved observation, the extension of a crack observed in Fig. 1 (c) did not occur during the propagation of stress waves at the crack [10]. This means that our previous interpretation on the crack extension based on the interference of stress waves was not correct, so the mechanism of the crack extension has not been elucidated yet.

In this study, we observed the dynamics of fs laserinduced cracks after parallel fs laser irradiation inside a $\mathrm{LiF}$ single crystal to find the origin of the crack extension observed in Fig. 1 (c). We found fast and slow dynamics in the crack propagation and the slow dynamics were observed only in the extended cracks. In addition, transient birefringence distributions during crack propagation were observed using a pump-probe polarization microscope. Observation by a polarization microscope can visualize strain and stress distributions, because birefringence mainly comes from lattice deformation due to strain. From the observed transient birefringence distribution, we found that the strain, which was distributed around the unextended cracks, disappeared around the extended cracks.

\section{Methods}

The optical setup of a pump-probe microscope for the observation of crack dynamics is shown in Fig. 2. This optical setup consists of mainly two parts: one is a parallel fs laser irradiation system with a spatial light modulator (SLM) and the other is a pump-probe polarization microscope.

In the part of parallel laser irradiation, the spatial phase distribution of a pump pulse from a Ti-sapphire fs laser with regenerative amplifier (Coherent; Mira-Legend, wavelength was $800 \mathrm{~nm}$, pulse width was $120 \mathrm{fs}$.) was modulated when it was reflected on an SLM (Hamamatsu; X10468$02)$. The phase modulated pump pulse was focused inside a LiF single crystal with a $50 \times$ objective lens (focal length was $4 \mathrm{~mm}$ and NA was 0.80 ) after passing through a telescope (magnification was 0.6). The incident of the pump pulse was normal to the (001) surface of the LiF single crystal. Inside the LiF single crystal, fs laser pulse was focused at three spots and nonlinear photoexcitation occurred at each spot. To generate focal spots of a fs laser pulse at desired positions, the phase modulation pattern (phase hologram) was prepared by the numerical calculation based on the Optimal Rotation Angle method [11].

In the part of a pump-probe polarization microscope, a probe pulse (wavelength was $400 \mathrm{~nm}$; the second harmonic of a fs laser pulse of $800 \mathrm{~nm}$ ) was delayed relative to the pump pulse by an optical delay line and passed through the photoexcited region inside the LiF single crystal. Before the crystal, the probe pulse was made circularly polarized by a polarizer and quarter waveplate. After passing through the crystal, the probe pulse was passed through the objective lens, reflected by a dichroic mirror (DM) and the mag- nified transmission intensity distribution was detected by a charge coupled device (CCD) camera with an imaging intensifier (Hamamatsu; C10054-03). To analyze the polarization change of the probe pulse by a transient birefringence distribution due to laser-induced phenomena, a quarter waveplate and analyzer were placed in front of the CCD camera. By analyzing the intensity distributions of the probe pulse measured at various orientations of the quarter waveplate (QWP2) and analyzer (Polarizer 2), the birefringence distribution around the photoexcited region was obtained. The analysis of the birefringence was described in detail in our previous paper [12, 13].

When the dynamics after 10 ns was observed, we added a time delay of about $14.3 \mathrm{~ns}$ to the probe pulse by making an additional optical path. Because the maximum time delay of the optical delay line was $10 \mathrm{~ns}$, the maximum time delay of the pump-probe microscope was $24.3 \mathrm{~ns}$.

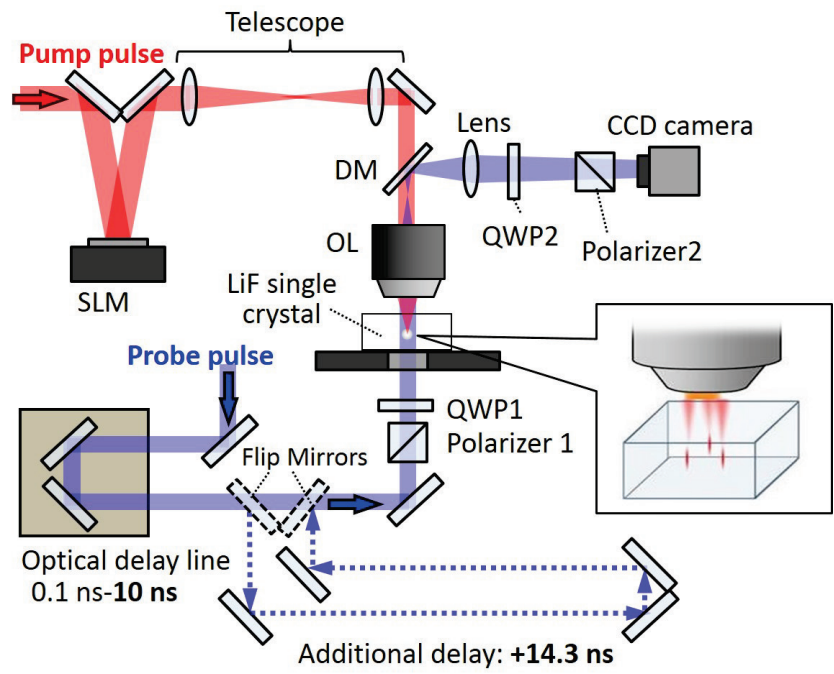

Fig. 2 Schematic of pump-probe polarization microscope for the observation of birefringence distribution after photoexcitation by a focused fs laser pulse at multiple spots inside a LiF single crystal. SLM: spatial light modulator; DM: dichroic mirror; QWP1, QWP2: quarter waveplates; OL: objective lens.

\section{Results}

\subsection{Cracks by parallel laser irradiation}

First, we investigated how the crack extension depends on the energy of a fs laser pulse and the distance between photoexcited regions. Figures 3 (a) and 3 (b) show transmission optical microscope images of fs laser-induced cracks after parallel photoexcitation at three spots inside a LiF single crystal. The three photoexcited spots were located at the corners of isosceles triangle, of which base is parallel to the [100] of the LiF single crystal. In this paper, we denote the distance between the upper and lower photoexcited points as $l$. Although the pulse energy at each spot was the same $(2 \mu \mathrm{J})$ both in the case of $l=20 \mu \mathrm{m}$ (Fig. 3(a)) and $l=30 \mu \mathrm{m}$ (Fig. 3(b)), the lengths of the cracks were different. In the case of $l=20 \mu \mathrm{m}$, the lengths of cracks were different apparently, whereas the lengths of all the cracks were almost the same in the case of $l=30 \mu \mathrm{m}$. This difference suggests that the interaction between three photoexcited regions was smaller when the distance between photoexcited regions was longer.

We focused the upward crack from the upper photoexcited region (Crack A) and the downward crack from the 
same photoexcited region (Crack B) and plotted the lengths of Crack A and Crack B against the pulse energies (Fig. 3 (c)). The length of Crack A increased as the pulse energy increased almost linearly. On the other hand, steep rises appeared in the length of Crack B. The pulse energy at the steep rise became larger as $l$ became longer and was almost proportional to $l$. This behavior of Crack B suggests that the influence from other photoexcited region to Crack B could decrease as increasing distances between three photoexcited regions.

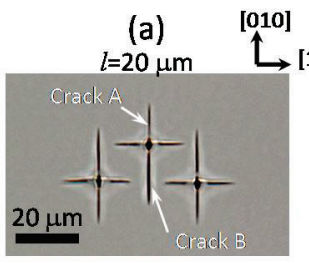

(b)
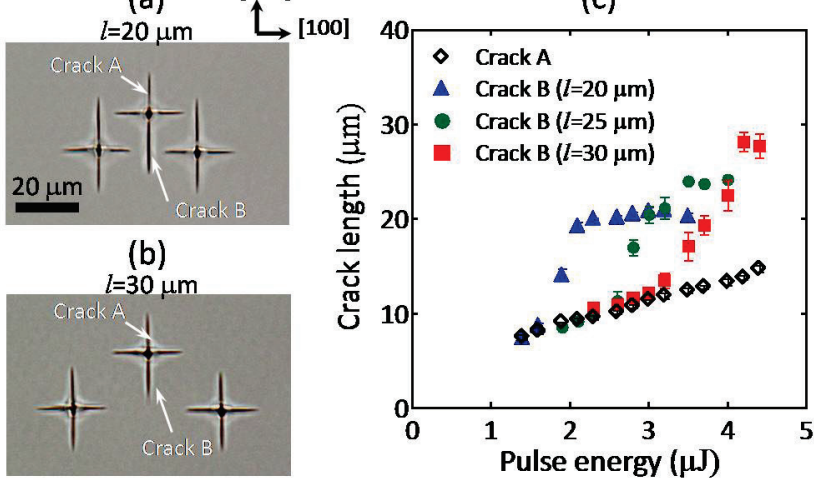

Fig. 3 (a), (b) Transmission optical microscope images of fs laserinduced cracks after simultaneous photoexcitation at three spots inside a $\mathrm{LiF}$ single crystal. The distances between the upper and lower photoexcited points were different, but the pulse energies were the same ( $2 \mu \mathrm{J}$ at each spot). The scale bar corresponds to 20 $\mu \mathrm{m}$. (c) Lengths of cracks plotted against the pulse energies.

\subsection{Crack propagation dynamics}

To elucidate the dynamics of Crack A and Crack B, transient transmission images after fs laser irradiation were observed by a pump-probe microscope without the Polarizer 2. Figure 4 shows transmission images around the Crack A and Crack B at various time delays after fs laser irradiation. In this observation, the distance between the upper and lower photoexcited points was $25 \mu \mathrm{m}(l=25 \mu \mathrm{m})$. The pulse energy was about $3 \mu \mathrm{J}$ at each spot. Four cracks were generated from the upper photoexcited region at $1 \mathrm{~ns}$ and they propagated at the same velocity until about $4 \mathrm{~ns}$. After $5 \mathrm{~ns}$, the length of Crack A did not changed, while Crack B propagated slowly until 24 ns. At 24 ns, the length of Crack $\mathrm{B}$ was about 1.6 times longer than that of Crack A. At $1 \mathrm{~s}$ after photoexcitation, at which crack elongation had already finished, three cracks, the downward crack from the upper photoexcited point and the two upward cracks from the lower photoexcited points, became more than 1.6 time longer than any other cracks.

The lengths of three cracks (Crack A, B, C) were plotted against the time delays in Fig. 5. The plot show that all the cracks propagated at the same velocity until $4 \mathrm{~ns}$, but additional propagation occurred in Crack B and Crack C. The velocity of the fast crack propagation until 4 ns was about $3.8 \mu \mathrm{m} / \mathrm{ns}$, which is comparable to the velocity of the transversal elastic wave in a LiF single crystal $(4.9 \mu \mathrm{m} / \mathrm{ns})$ [14]. On the other hand, the slower propagation velocity was more than ten times slower than that of the fast propagation and different between Crack B and Crack C; the velocity of Crack B was about $0.45 \mu \mathrm{m} / \mathrm{ns}$, while that of Crack $\mathrm{C}$ was about $0.25 \mu \mathrm{m} / \mathrm{ns}$. Because the slower propagation occurred after the stress waves propagated away completely from the regions of cracks, there could be some stress that induced slower dynamics.

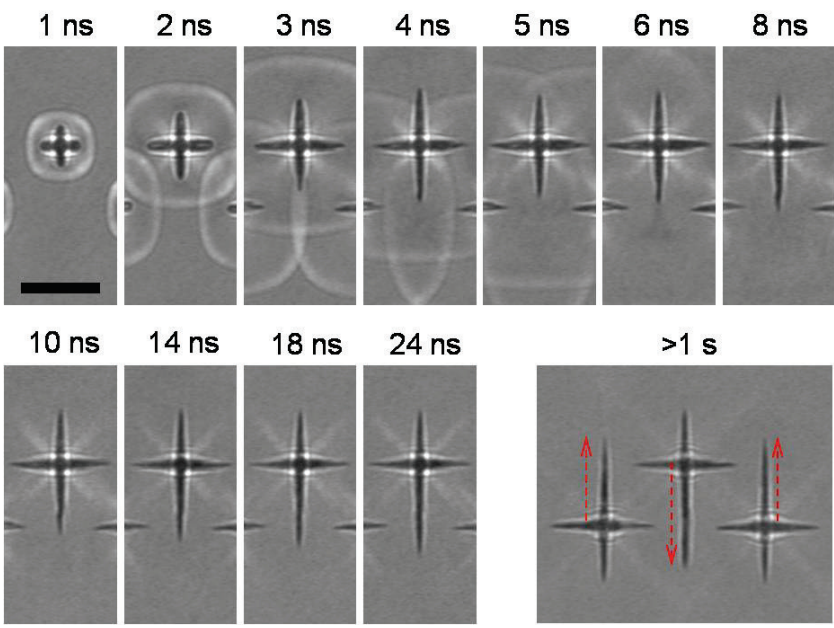

Fig. 4 Transmission images of fs laser-induced cracks at various time delays after simultaneous photoexcitation at three spots inside a LiF single crystal. The scale bar corresponds to $20 \mu \mathrm{m}$.

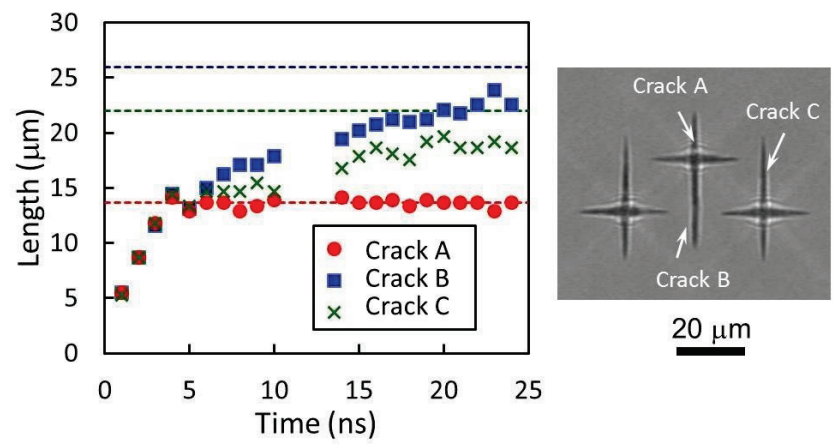

Fig. 5 Lengths of cracks plotted against the time delays. Broken lines indicate the final crack lengths measured by the transmission optical microscope image at $>1 \mathrm{~s}$.

\subsection{Dynamics of strain distribution}

To elucidate the stress that induced slower propagations of Crack B and Crack C, the transient birefringence distributions after photoexcitation were observed by a pumpprobe polarization microscope. Figure 6 (a) shows transient birefringence distributions at various time delays around the three photoexcited regions inside a LiF single crystal. The pulse energy and the positions of the three photoexcited regions were the same as those in the observation of Fig. 4. The observed birefringence distributions reflect the temporal evolution of the strain and stress distributions. The strain distribution around the cracks varied drastically by interference of laser-induced stress waves until $6.0 \mathrm{~ns}$. Because the slowest component of stress waves in a LiF single crystal is the quasi-transverse elastic wave that propagates in $\langle 100\rangle$ and the velocity is $4.9 \mu \mathrm{m} / \mathrm{ns}$, the influence by the stress waves can be negligible in the regions of cracks after $8 \mathrm{~ns}$. After $8 \mathrm{~ns}$, birefringent regions extended in four $<110>$ directions from each photoexcited regions remained and they remained at $24.3 \mathrm{~ns}$.

These transient birefringent regions in the $<110>$ directions were also observed in the case of single spot's fs laser irradiation [Fig. 6 (b)]. The slow axis of the birefringent region is parallel to the extended direction, i.e. radial direction from the photoexcited region. This slow axis means 
that the crystal in the birefringent region was compressed in the radial direction. Because the compression in the radial direction can make dislocations and long dislocation bands are generated finally after fs laser irradiation (Fig. 1 (a)), the birefringent regions should be attributed to strain by dislocations distributed around the $<110>$ lines.

(a)

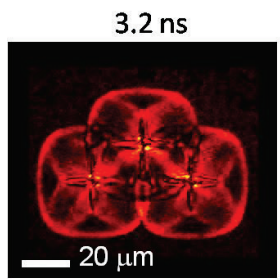

$8.0 \mathrm{~ns}$

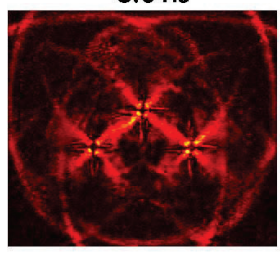

$19.3 \mathrm{~ns}$
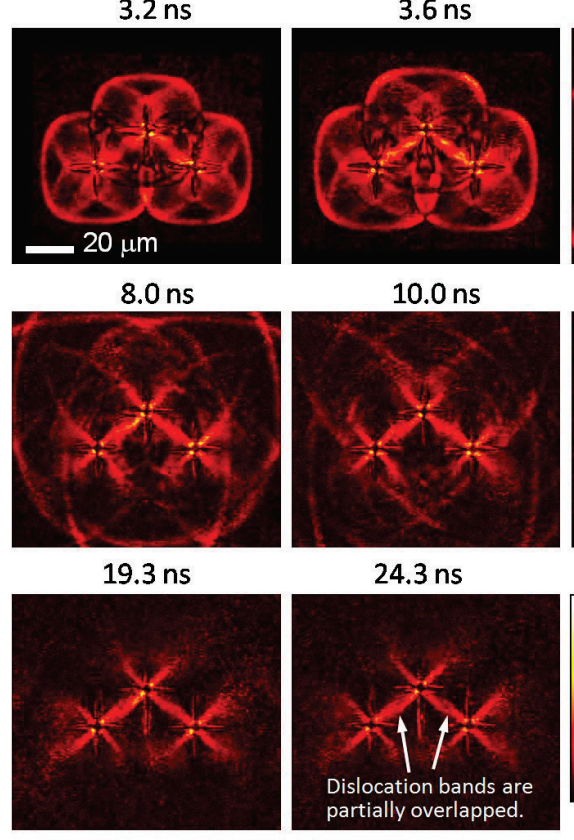
$10.0 \mathrm{~ns}$

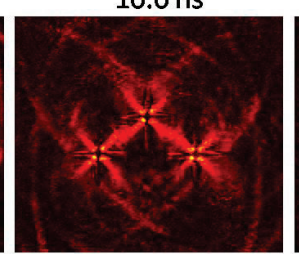

$24.3 \mathrm{~ns}$
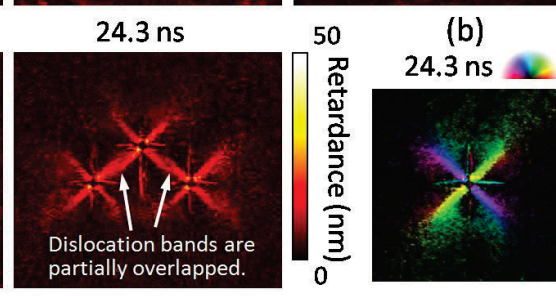

Fig. 6 (a) Distributions of retardance of transient birefringence at various time delays after photoexcitation at 3 spots inside a $\mathrm{LiF}$ single crystal. The scale bar corresponds to $20 \mu \mathrm{m}$. (b) Birefringence distribution at $24.3 \mathrm{~ns}$ after fs laser irradiation at a single spot. The color indicates the slow axis of the birefringence.
Retardance at $24.3 \mathrm{~ns}$
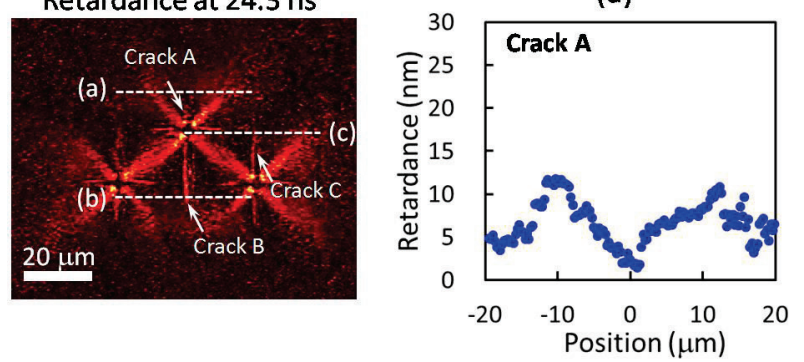

(b)

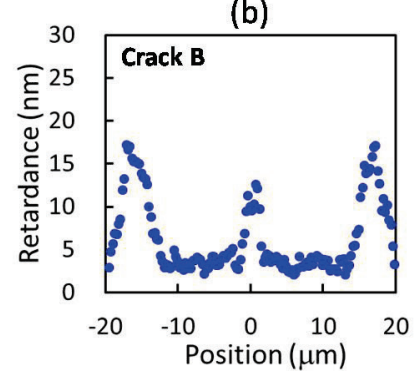

(c)

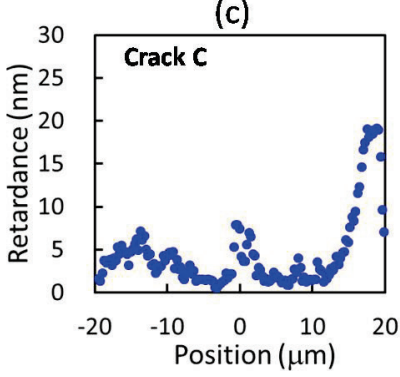

Fig. 7 Retardance of birefringence at 24.3 ns plotted against the positions on the lines which pass the tips of Crack A, B and C. The lines are drawn in the retardance image at $24.3 \mathrm{~ns}$.

To compare the strain distributions around Crack A, B and $\mathrm{C}$, the retardance (amplitude of birefringence) distributions near the crack tips at 24.3 ns were plotted in Fig. 7. Birefringence was distributed clearly in a wide area around the tip of a normal crack, Crack A (Fig. 7(a)), which means that strain was loaded on the tip of the crack. On the other hand, around the tips of the extended cracks, Crack B and C (Fig. 7 (b) and 7 (c)), the birefringence was flat and smaller than $3 \mathrm{~nm}$, which is comparable to the error level in the analysis . The small birefringence around Crack B and $\mathrm{C}$ means that there was little strain around Crack B and C. In terms of the observed birefringence around the cracks and the difference between Crack A, B and C, we interpreted that the transient strain around the tip of a crack tip prevented slow dynamics in Crack A, while the slow propagation dynamics appeared in Crack B and $\mathrm{C}$ due to the disappearance of the strain around the cracks.

The origins of the strain around Crack A and the driving force of a slow propagation dynamics still remain unknown. The origin of the strain around Crack A should be strain field by dislocation bands along $<110>$. According to the dislocation theory [15], the strain and stress fields are generated around a dislocation, because there is a lattice mismatch at a dislocation. Because the stress amplitude of a dislocation is proportional to the inverse of the distance, the stress could generate enough large strain around neighbour cracks and prevent the cracks from propagating further. However, the strain due to dislocation bands could become smaller when multiple dislocation bands of negative directions are located closely. In the case of parallel photoexcitation at 3 spots, two dislocation bands generated from two photoexcited regions are partially overlapped (24.3 ns in Fig. 6 (a)). Because these two dislocation bands are directed in the opposite directions, the strain near the nearly overlapped dislocation bands could be cancelled out. Therefore, we speculated that the small birefringence around Crack B and Crack C could be attributed to the strain decreased due to partially overlapped dislocation bands

One of the possible origins of the slow crack propagation is thermal stress in the photoexcited region. Because thermal diffusion occurs after several tens nanoseconds, thermal stress of enough large amplitude still remained during slow crack propagation. According to the observation of fs laser-induced stress wave, the temperature change in the photoexcited region must be higher than $1000 \mathrm{~K}[16$, 17]. The thermal stress $\left(P_{\text {thermal }}\right)$ can be calculated by

$$
\mathrm{P}_{\text {thermal }}=\left(\mathrm{C}_{11}+2 \mathrm{C}_{12}\right) \beta \Delta \mathrm{T}
$$

where $C_{11}$ and $C_{12}$ are the elastic constants, $\beta$ is thermal expansion coefficient, and $\Delta \mathrm{T}$ is temperature change [18]. Using $\mathrm{C}_{11}=111 \mathrm{GPa}$, and $\mathrm{C}_{12}=42 \mathrm{GPa}$ and $\beta=3.3 \times 10^{-5}$ of a $\mathrm{LiF}$ single crystal $[19,20]$, the thermal stress of $\Delta \mathrm{T}=1000$ $\mathrm{K}$ is $6.4 \mathrm{GPa}$. Although this stress is relaxed partially due to a stress wave generation, enough large stress could remain after the stress wave propagation and the remained stress possibly could induce further crack propagation.

\section{Conclusion}

The fast and slow propagation dynamics were observed in fs laser-induced cracks after parallel fs laser irradiation inside a LiF single crystal. The transient birefringence imaging during the slow crack propagation showed that strain remained around the normal cracks (the cracks of no slow propagation), whereas the strain around the extended cracks (with slow propagation) was much smaller. Therefore, we interpreted that the slow crack propagation appeared in the region where the strain around the crack be- 
came smaller due to the interaction between dislocation bands around the crack. Therefore, the results shown in this paper suggest that the prediction of crack generation in fs laser processing inside single crystals needs the consideration on the transient strain distributions around cracks due to laser-induced dislocations.

\section{Acknowledgments}

This research was supported partially by Japan Society for the Promotion of Science (JSPS) Grant-in-Aid for Scientific Research (C), No. 26410240 and the CSTI, SIP (Innovative design/manufacturing technologies) of NEDO of Japan.

\section{References}

[1] K. M. Davis, K. Miura, N. Sugimoto, and K. Hirao: Opt. Lett., 21, (1996) 1729.

[2] E. N. Glezer, M. Milosavljevic, L. Huang, R. J. Finlay, T.-H. Her, J. P. Callan, and E. Mazur: Opt. Lett., 21, (1996) 2023.

[3] A. Vogel, J. Noack, G. Huttman, G. Paltauf: Appl. Phys. B, 81, (2005) 1015.

[4] S. Kanehira, K. Miura, K. Fujita, K. Hirao, J. Si, N. Shibata, Y. Ikuhara: Appl. Phys. Lett., 90, (2007) 163110.

[5] B. Qian, J. Song, G. Dong, L. Su, B. Zhu, X. Liu, S. Sun, Q. Zhang, J. Qiu: Opt. Express, 17, (2009) 8552.

[6] M. Sakakura, T. Tochio, M. Eida, Y. Shimotsuma, S. Kanehira, M. Nishi, K. Miura, K. Hirao: Opt. Express, 19, (2011) 17780.
[7] S. Juodkazis, K. Nishimura, S. Tanaka, H. Misawa, E. G. Gamaly, B. Luther-Davies, L. Hallo, P. Nicolai, V. T. Tikhonchuk: Phys. Rev. Lett., 96, (2006) 166101.

[8] M. Sakakura, Y. Ishiguro, N. Fukuda, Y. Shimotsuma, and K. Miura: Opt. Express, 21, (2013) 26921.

[9] M. Sakakura, Y. Ishiguro, N. Fukuda, Y. Shimotsuma, and K. Miura: Appl. Phy. A, 114, (2014) 261.

[10] M. Sakakura, N. Fukuda, Y. Shimotsuma, and K. Miura: Proc. SPIE, 8967, (2014) 89670C.

[11] J. Bengtsson: Appl. Opt., 33, (1994) 6879.

[12] T. Tochio, M. Sakakura, Y. Shimotsuma, M. Nishi, K. Hirao, and K. Miura: Jpn. J.Appl. Phys., 51, (2012) 126602.

[13] M. Sakakura, T. Tochio, Y. Ishiguro, M. Nakabayashi, Y. Shimotsuma, K. Hirao, K. Miura: Proc. SPIE 8530, (2012) 853003

[14] M. J. Musgrave: Rep. Prog. Phys., 22, (1959) 74.

[15] M. Kato: "Introduction to the theory of dislocations" (Shokabo, Tokyo, 1999) Chapter 3.

[16] M. Sakakura, M. Terazima, Y. Shimotsuma, K. Miura, and K. Hirao: Opt. Express, 15, (2007) 5674.

[17] M. Sakakura, M. Terazima, Y. Shimotsuma, K. Miura, and K. Hirao: Opt. Express, 15, (2007) 16800.

[18] L. D. Landau and E. M. Lifshitz: "Theory of elasticity" (Pergamon, Oxford, 1986) Chapter VI.

[19] C. V. Briscoe, and C. F. Squire: Phys. Rev. 106, (1957) 1175.

[20] S. Lambade, G. Sahasrabudhe, and S. Rajagopalan: J. Appl. Phys., 78, (1995) 6525.

(Received: May 25, 2015, Accepted: December 7, 2015) 